Kalpa Publications in Computing
Volume 12, 2019, Pages 241-249
Proceedings of 4th International Conference on the
Internet, Cyber Security and Information Systems 2019

\title{
Privacy Concerns of Overweight Adults to use Wearable Devices for Sustained Health Monitoring
}

\author{
Oluwaseyi C. Ogundele ${ }^{1}$ and Liezel Cilliers ${ }^{2}$ \\ Information Systems Department, Faculty of Management \& Commerce, University \\ of Fort Hare, East London, South Africa \\ seyics@gmail.com, lcilliers@ufh.ac.za
}

\begin{abstract}
The market for wearable devices that can be used for sustained health monitoring purposes is continuously growing within the healthcare sector. However, to function effectively, these devices must collect a large amount of data from the users. There are privacy concerns that may inhibit the behavioural intention of overweight adult to use wearable devices for health monitoring in the long term. This study examined the privacy factors influencing the behavioural intention of overweight adult to make use of wearable devices of sustained health monitoring. The study made use of a qualitative research approach with an interview design. A purposive sampling technique was used to select and interview twenty overweight adults (aged 18-59 years) who are using wearable devices in East London, South Africa. The Expectation Confirmation Model $(E C M)$ framework was adopted as the underlying research theory in this study. Thematic analysis was used to analyse the data provided by participants. The results found that there were 4 levels of privacy concerns among users. Some users were very concerned that their data was collected by the device manufacturing, while others had not concern at all. Some users had privacy concerns, but did not think that the data collected would be useful to a third party and finally some users did have privacy concerns, but indicated that the benefit of using a wearable device outweighed their concerns and they would continue to use the device. The recommendation of the study is that users must educate themselves about what data is collected and how it will be used by third parties.
\end{abstract}

Keywords: obese adults, wearable devices, data privacy, wearable devices, sustained monitoring

\section{Introduction}

The amount of overweight people worldwide has doubled over the last three to four decades (Omran, 2017; Seidell, \& Halberstadt, 2015). Statistics show that the prevalence of overweight adults 
have increased in South Africa with over $70 \%$ of women and $23.3 \%$ of men being considered obese (The Heart and Stroke Foundation, 2016). Being overweight is a condition that is characterised by a person having excess accumulated body fat (Nichols, 2016). According to the National Department of Health South Africa [NDoH] (2015: p.21), "Overweight and obesity reflects a gain of excess body fat, resulting from the cumulative and initially unnoticeable effects of day-to-day eating and physical activity behaviours that create a surplus of energy consumed relative to expended". Being overweight has been found to increase the risk of developing joint disease, stroke, type 2 diabetes, heart diseases, hypertension, depression, and in some cases, cancers (NDoH, 2016).

Self-monitoring has been identified as a key determinant in the management of weight loss (Perenc, Radochonska, \& Zaborniak-Sobczak, 2015). The aim of health monitoring is to help patients take responsibility for their health. Sustained monitoring involves an increased knowledge about diet and activity levels for effective decision-making related to weight control. Findings from previous studies have suggested that sustained self-monitoring is a vital element of treating overweight adults (Rosenbaum, Clark, Convertino, Call, Forman, \& Butryn, 2018; Perenc et al., 2015; Asch, Muller, \& Volpp, 2012).

Wearable medical devices are an effective way to ensure preventative health monitoring of weight at an initial stage in order to improve patients' activity levels (Zheng, Shen, Zhang, Wu, Zhang, \& Lu, 2013). According to Kaewkannate, and Kim (2016), "a wearable device is a new type of technology in the form of small hardware that includes an application with tracking and monitoring fitness metrics such as distance walked or run, calories consumed, and in some devices heart rate and sleep tracking" (p. 1). Wearable devices have a positive impact on overweight adults' health and wellness, offering a possible solution to improving their activity levels (Kelley, 2014).

Wearable devices allow self-monitoring of patients' behaviour on a daily basis from which decisions about their health are usually made to enhance their lifestyle Asch et al., 2012). These devices must collect a large amount of data from users in order to be effective which introduces privacy concerns (Cilliers, 2019). There are two types of data collection that is needed for users to keep track of their health. The first is automatic data collection using sensors by the wearable device and the second is where users must manually enter the data into the device. Examples of automatic data collection include GPS location; activity/movements and sleep patterns; blood pressure, blood oxygen levels and heart rate. Manual data entry is needed for food consumption and mood information (Cilliers, 2019). All this information can be shared via the Internet with healthcare providers to make informed decisions about the user's healthcare (Ngamntwini, \& Cilliers, 2018).

Confidentiality and security of personal data collected by wearable devices are major issues faced by users of wearable devices (Al-Azwani, \& Aziz, 2016). Health information is regarded as the most confidential of all types of personal information (Mehraeen, Ghazisaeedi, Farzi et al., 2016). Privacy is defined as "the claim of individuals, groups, or institutions to determine for themselves when, how, and to what extent information about them is communicated to others" (Westin, 1967: 2). There is a concern from users that the device manufacturer will share their data with third parties which is responsible for the analysis of the data (Al-Azwani et al., 2016). These include behaviour, gender, profiles, age, location, social media accounts and email address as well as GPS information. Thus, users of these devices are worried about data sharing and confidentiality of their information with the manufacturers because they do not own their data (Spann, 2016). Fiercebiotech (2014) found privacy concerns is a barrier that prevent the continuous use of wearable devices. 
Privacy and security are factors that will influence a user's intention to make use of wearable devices to monitor their health (Pfeiffer, Entress-Fürsteneck, Urbach, \& Buchwald, 2016; Mottim, \& Caine, 2015). This is similar to the findings of DuFour, Lajeunesse, Pipada and Xu (2016) who examined users' levels of concern about data privacy, and how these concerns will affect the intention to use wearable fitness trackers. Acquisti, Gross and Stutzman (2011) identified privacy and security as part of the five top vital concerns that users consider important in the wearable technology continuous usage. Users felt they buy wearable devices and generate the data that manufacturers of wearable devices collect, store and later sell to a third party (Piwek, Ellis, Andrews, \& Joinson, 2016). PricewaterhouseCoopers [PWC] (2016) found that $86 \%$ of users showed serious concerns about the privacy of wearable devices which also influenced their intention to use the wearable device. These concerns included theft of data from the device by unauthorised parties, theft of data generated by wearable devices that are stored in cloud networks (Popat, \& Sharma, 2013). This study examined the privacy factors influencing the behavioural intention of overweight adult to make use of wearable devices of sustained health monitoring.

The Expectation Confirmation Model (ECM) framework developed by Bhattacherjee (2001b) was adopted as the underlying research theory in this study. The ECM is mostly centred on postacceptance elements because it postulates that the outcome of any post acceptance elements are already accomplished within the satisfaction and confirmation elements (Bhattacherjee, 2001a). In application to this study, the use of ECM provides a better knowledge and understanding of the privacy concerns that will influence overweight adult's intentions to use wearable devices for sustained health monitoring.

\section{Methodology}

This study adopted a qualitative research approach. The population that was used for this research project were overweight adults who are users of fitness tracking devices in East London, South Africa. The sample size was 20 overweight adults and users of fitness tracking devices ranging from 18 59 years of age. Whether the adults were overweight was determined by using the respondents' body mass index (BMI). The World Health Organisation guidelines state that people with a BMI between $25 \mathrm{~kg} / \mathrm{m} 2$ and $29.9 \mathrm{~kg} / \mathrm{m} 2$ can be considered to be overweight (World Health Organisation [WHO], 2016). The researcher employed a purposive sampling technique to identify the overweight participants. A semi-structured interview was used to obtain deep insights on how overweight adults' behavioural intention to make use of wearable devices can be influenced for sustained health monitoring. The interviews were transcribed and a thematic analysis method was adopted in this study. The researcher received ethical clearance approval from the University Research Ethics Committee. The privacy factors influencing the behavioural intention of overweight adult to make use of wearable devices for sustained health monitoring is discussed in the next section of this study.

\section{$3 \quad$ Results}

This study examined the privacy factors influencing the behavioural intention of overweight adult to make use of wearable devices of sustained health monitoring. Twenty overweight adults and users of wearable devices between the ages of $18-59$ years, living in East London, South Africa was interviewed. Eight of the participants were male; while 12 were female. Three of the participants were 
between 18-25 years; 9 of the participants were between 26-35 years; 5 of the participants were between 36-50 years while 3 of the participants were between 51-59 years. The average Body Mass Index (BMI) of the participants was 27.91; while the minimum was 25.20 (Participant thirteen) and the maximum was 29.4 (Participant nineteen). This indicated that all the participants were overweight adults. The most common wearable devices among the participants were Samsung Gear fit used by 4 participants, Apple iWatch used by 4 participants, while 4 participants used Garmin. Four participants used Nike + and Fitbit respectively, while the remaining 4 participants used Solace, Huawei TalkBand B3, Polar, and Bfit.

As noted by Herring (2016), privacy is the ability of a person to conceal his/her vital information. In the context of this study, privacy setting is used as a means to conceal vital information of the users of wearable devices. Two themes were identified during the interview process: 1) Privacy affecting the intention to use wearable devices and 2) privacy and control over information.

\subsection{Theme 1: Privacy affecting the intention to use wearable devices}

Grand View Research (2016) submitted that privacy issues are one of the major challenges in wearable devices which are influencing behavioural intention of users and in the next few years, may affect the growth of wearable technology. Boscart et al (2008) reported that users' insecurity about their privacy was a significant factor in the continuous usage of the wearable device. The study of Acquisti et al (2011) identified privacy and security as part of the five top vital concerns that users considered important in the continuous usage of wearable technology. Furthermore, Sconce (2016) submitted that mistrust of wearable technology by users posed a serious challenge to their behavioural intention as a result of data insecurity (DuFour et al., 2016; Popat et al., 2013).

There were four levels of concern that was identified during the interviews. The first level of concern was the most serious where the user indicated that they will stop using the wearable device due to privacy concerns. Participant twenty raised serious privacy concerns when using wearable devices. The participant stressed that he feared that his vital signs information might be leaked to unauthorised parties. This agreed with findings of (Al-Azwani et al 2016; Spann 2016; Salah, MacIntosh, \& Rajakulendran, 2014). Salah et al (2014) found that users of wearable devices were more concerned about their health information. Also, Spann (2016) reported that the users of wearable devices feared that their vital information might fall into the wrong hands. When the participant was asked whether this could hinder his behavioural intention to continue using wearable devices for sustained health monitoring, the participant noted that "it can hinder my continuous use of the device because I will stop the usage of the device once I notice any form of infringements on my personal information stored on the device". He added that he might not advise other people to use wearable devices for sustained health monitoring because their health information might be easily tampered with by unauthorised persons (Participant twenty). From this it could be deduced that privacy issues could negatively affect the behavioural intention of some users of wearable devices.

The second level of concern points to privacy concerns amongst the users, but that the concern did not outweigh the benefit of using the device. Two participants reported moderate privacy concerns during the data collection stage of the study. When the researcher asked whether the concern for privacy could hinder their continuous usage of wearable device, the participant sixteen responded as follow:

"it would definitely affect it because it would not be fine if he discovered that someone was having access to what was supposed to be private to him" (Participant sixteen) 
"privacy cannot hinder me from the continuous use of the devices due to the benefit I'm gaining from the usage of the device" (Participant one).

As noted by Spann (2016), the users of wearable devices are concerned about sharing their information with third parties. Salah et al (2014) stressed that privacy and security are of paramount concern, especially with wearable devices that collect health and medical information. These fears related to wearable devices are posing a threat to users' continuous intention (Salah et al., 2014). When the two participants was probed further if they would still recommend wearable devices to someone despite the privacy concerns they had pointed out, the participants expressed that they would warn others not to use the device if they had privacy concerns.

"If they don't mind their privacy loss, because I'm looking at the benefit that they are going to derive. You will notice that sometimes you might have to forgo your privacy if your condition means you have to do it. So it is a matter of weighing up how much you are going to lose and what you are going to gain at the end of the day. So if my benefits are more I would rather recommend it to other people in as much you going to lose ABC and D in form of privacy lose. Sometimes it's worth it to lose your privacy to be healthy. At the end of the day, inasmuch as the device is taking our health information, we are gaining" (Participant sixteen).

This agreed with the submission of Al-Azwani et al (2016) when they submitted that the producers of wearable devices gathered data from users' and there were notions that some of these producers were willing to share users' information with third parties. This information sharing would include behaviour, gender, profiles, age, location, social media accounts and email address as well as GPS information. Confidentiality of personal data produced by the users is thus a major issue of wearable devices (Al-Azwani et al., 2016).

The third level of concern was that the data collected was not sensitive, so privacy was not an issue to the participants. A male participant initially stated that he was concerned about his privacy because he did not know where his information went and what it might be used for in the future. He said a hacker could easily gain access to the database of the companies that produced wearable devices and retrieve all the information. He feared that his privacy was at stake because somebody could easily use his information against him in the future (Participant seven). Nonetheless, in the later part of the interview, he stated that "I don't care if anyone can see, maybe they will be motivated to get one to monitor their exercise; I don't think it poses any security threat". This participant felt that when people saw the numerous functions of the device in terms of sustained health monitoring, they might be motivated to get one for themselves too.

The last level of concern was where five participants emphasised that they were not concerned about privacy. For instance, participant three noted that she was not even worried about her privacy. She said she would only be worried when something happened to her. According to her, "privacy is not one of my priorities. It is hardly something I even think about". Also, participant twelve said "what will anyone have to do with my wearable device history?" The participant noted that it is of no use to them. Similarly, participant thirteen stressed that "why would I have privacy concerns? I'm not concerned about sharing my wearable device history with the third parties because who else needs to know about my heart rate and my vital signs if it is not the third parties"? Also, a participant expressed that "not really, I don't think the data it collects is important enough to steal or will invade my privacy" (Participant nineteen). Also, a participant noted that "I don't feel it's a private matter. I don't care if my information is seen by someone else. Honestly I'm not that kind of private person, it is not like it is a secret thing like HIV status. It is just my work out histories, heart beat histories" (Partici- 
pant fifteen). It can be deduced from the above narratives that the participants argued that their vital signs could not be used against them by the third parties. They (third parties) could only use those vital signs to motivate themselves to also start using wearable devices for sustained health monitoring. Thus, the participants under this sub-theme were not concerned about making her information known to third parties. Nonetheless, a participant emphasized that she was not so worried about privacy because of the information revolution. She said "I am not worried about privacy because we are in the age where everything is online. I'm sure the security is not a problem" (Participant ten). When the researcher asked whether she would continue the usage of wearable devices for sustained health monitoring even if her vital signs were exposed to the public, she replied that she could not say for then. She said she was still doing her research on the issue of privacy. The result of her research would determine her future action on the continuous usage of wearable devices (Participant ten). Also, a participant stressed that he could decide with whom he wanted to send or share his information.

\subsection{Theme 2: Privacy and control over information}

Five out of the twenty participants were not concerned about privacy while using wearable devices because they could control access to their vital signs. For instance, participant two noted that "people can only see my steps for the day or the week. But only I can see my heart rate and my stress levels" (Participant two). This implies that the participant had control over who could have access to her vital signs. This could mean that there is a function in the wearable device where users can control access to their vital signs. Also, a participant stressed that she could control access to her information while using wearable devices. She expressed that "I can block access to my information. So, if I want to link it to my Apps, I can link it and share my activities with other people, but if I don't want to, no one can access it" (Participant eighteen).

Participant eighteen added a new perspective to this sub-theme when she expressed that it should be something enjoyable and exciting sharing your information with others to see. The participant said she felt excited sharing her information with other people so that they knew the importance of wearable device in health monitoring. This is an indication that the participant was satisfied with the wearable device for sustained health monitoring. This is in tandem with the expectation-confirmation model adopted in this study (Weng, Zailania, Iranmanesh, \& Hyun, 2017; Luqman, Razak, \& Ismail, 2014; Zheng et al., 2013). Participant six explained that there is a function in the wearable devices where you can share information. The participant expressed that you can only make it public if you want to. However, another participant disagreed that the wearable device could share information. He said that it could only share information with a paired App, which meant that users could decide whether they wanted to share information or not. He explained thus:

"Not really, because the device does not share information with anything rather than a paired App. You can only pair it with an App to share information and the App I'm using does not share information with anything. So again it is very limited and even if I had more advanced wearable device and I was using let my medical aid system. When I meet with my GP, I'm the one who said fine you can put me onto the program so if I now get an App that can pair, the App will pair with their program and the only one who should have access to that information is my medical practitioner. I'm not really concern about because if I walk around people can see that I'm overweight. So I'm not really worried about people knowing how much I'm weighing now and how high my weight is. The only concern might be discovery might use my detail for something like not allowing me to take certain insurance, something like that, but again, my insurance is with another company and not with Discovery" (Participant nine). 


\subsection{Summary of privacy concerns}

In summary, the narratives on the concerns for privacy in the use of the wearable devices among the overweight adults are nuanced. Some participants were of the view that their information could not be used by the third parties because it was only about their physical activities. They posited that their information about the number of calories burnt, number of steps they took while running and their heart rate were of no use to the third parties. On the other hand, some participants expressed that they had serious security and privacy concerns in the usage of wearable devices for sustained health monitoring.

Based on these narratives, the researcher deduced that some users might not have known that their wearable devices had options where they could choose whether to share their information or not. Also, the researcher implied that some users might not have been using the kind of wearable devices that have options to protect their vital signs (information). Additionally, the researcher inferred that some users might not know that sharing information through wearable devices required paired Apps. Therefore, based on these summations, it could be argued that with constant sensitisation of the users on the different functions that wearable devices have could help in ensuring that they safeguarded their vital signs by using functions for that purpose.

\section{Conclusion}

The studies of these scholars showed when the users of wearable devices found them efficient or they were satisfied with the devices in monitoring their health, their behavioural intention to continue the usage of the devices for sustained health monitoring would increase. Based on the results above, the privacy concerns were fourfold: users were not worried about their privacy; users would stop using the device due to the privacy concerned, those that had no privacy concerns as they did not think the data was useful and those that had privacy concerns but the benefits the device produced outweighed the concerns. Despite the above participants raised privacy concerns, they still emphasised they would continue using wearable devices for sustained health monitoring. This implies that these participants confirmed the perceived usefulness of the wearable devices for sustained health monitoring. The limitation of the paper is that only 20 people were interviewed which limits the generalizability of the results. The sample also included only participants between the ages of In future the population sample should be increased and included.

\section{Acknowledgements}

This research project was jointly funded by the South African Medical Research Council (SAMRC) and Forte, the Swedish Research Council for Welfare, Working Life and Welfare.

\section{References}

Acquisti, A., Gross, R., \& Stutzman, F. (2011). Faces of Facebook: Privacy in the age of augmented reality. Las Vegas: BlackHat.

Al-Azwani, I.K., \& Aziz, H.A. (2016). Integration of Wearable Technologies into Patients' Electronic Medical Records. Quality in Primary Care, 24(4), 151-155. 
Asch, D.A., Muller, R.W., \& Volpp, K.G. (2012). Automated hovering in health care-watching over the 5000 hours. The New England Journal of Medicine, 367(1), 1-3.

Bhattacherjee, A. (2001b). Understanding information systems continuance: An expectationconfirmation model. MIS Quarterly, 25(3), 351-370.

Bhattacherjee, A. (2001a). An empirical analysis of the antecedents of electronic commerce service continuance. Decision Support Systems, 32 (2), 201-14.

Boscart, V., McGilton, K., Levchenko, A., Hufton, G., Hollidayand, P., \& Fernie, G. (2008). Acceptability of a wearable hand hygiene device with monitoring capabilities. Journal of Hospital Infection, 70, 216-222.

Cilliers, L. (2019). Wearable devices in healthcare: Privacy and information security issues. Health Information Management Journal, 1833358319851684.

DuFour, A., Lajeunesse, K., Pipada, R. \& Xu, S. (2016). The effect of data security perception on wearable device acceptance: A Technology Acceptance Model. Retrieved September 18, 2017, from http://csis.pace.edu/ ctappert/srd2017/2016fallproj/d4t06.pdf

Fiercebiotech. (2014). Report: Wearable devices held back by privacy and cost concerns. Retrieved June 20, 2017, from https://www.fiercebiotech.com/medical-devices/report-wearabledevices-held-back-by-privacy-and-cost-concerns

Grand View Research (2016). Digital patient monitoring devices market size \& forecast by type (wireless sensor technology, mhealth, telehealth, wearable devices, remote patient monitoring), by product (diagnostic, therapeutic) and trend analysis from 2012 to 2022. Digital patient monitoring Devices Market Report, 2022. San Francisco, United States: Grand View Research. Retrieved October 10, 2017, from

Herring, J. (2016). Medical \& Law. (7th ed.). Oxford: Oxford University Press.

Kaewkannate, K., \& Kim, S. (2016). A comparison of wearable fitness devices. BMC Public Health, 16,433. DOI 10.1186/s12889-016-3059-0

Kelley, M.C. (2014). The impact of fitness technology on health outcomes. CMC senior theses. Paper 917. Retrieved from http://scholarship.claremont.edu/cmc theses/917

Luqman, A., Razak, R.C., \& Ismail, M. (2014). A review on mobile commerce continuance intention studies and its underlying model. Journal of basic applied scientific research, 4 (30), 1-9.

Mehraeen, E., Ghazisaeedi, M., Farzi, J., et al. (2016) Security challenges in healthcare cloud computing: a systematic review. Global Journal of Health Science 9(3): 157.

Mottim V.G., \& Caine, K. (2015). Users' privacy concerns about wearables: impact of form factor, sensors and type of data collected. Retrieved June 15, 2017, from http://fc15.ifca.ai/preproceedings/wearable/paper 2.pdf

National Department of Health, South Africa (2015). Strategy for the Prevention \& Control of Obesity in South Africa 2015 - 2020. Retrieved April 14, 2017, from http://cdn.24.co.za/files/Cms/General/d/4776/e9776ca8f6a44f3099dbde892bb0afbb.pdf

Ngamntwini, B. \& Cilliers, L. (2018). A usability framework for diabetic health applications in South Africa. In conference proceedings of Future Technology Conference 2018, 13-14 November 2018, Vancouver, Canada.

Nichols H. (2016). What is obesity? Medical news today. Retrieved March 15, 2017, from http://www.medicalnewstoday.com/info/obesity/

Omran, Z. (2017). Obesity: Current treatment \& future horizons. Sharjah, Bentham Science Publisher. Mini Review in Medicinal Chemistry, 17(1), 51 - 61 (11).

Perenc, L., Radochońska A., \& Zaborniak-Sobczak M. (2015). Role of selected self-monitoring techniques in treatment of overweight and obesity in adolescents. Medical Review 2015; 13(4), 387393. doi:10.15584/medrev.2015.4.6

Pfeiffer, J. Entress-Fürsteneck, M., Urbach, N., \& Buchwald, A. (2016). Quantify-me: consumer Acceptance of wearable self-tracking devices. Twenty-Fourth European Conference on Information Systems (ECIS), 12 - 15 June, 2016, Istanbul, Turkey. Research Papers. 99. 
Piwek, L., Ellis, D.A., Andrews, S., \& Joinson, A. (2016). The Rise of Consumer Health Wearables: Promises and Barriers. PLoS Medicine, 13(2). Retrieved June 20, 2017, from https://doi.org/10.1371/journal.pmed.1001953

PricewaterhouseCoopers (PWC). (2014). Health wearables: Early days. PricewaterhouseCoopers. Retrieved June 15, 2017, from https:/www.pwc.com/us/en/health-industries/top-health-industryissues/assets/pwc-hri-wearable-devices.pdf

Popat, K.A., \& Sharma, P. (2013) Wearable computer applications: A future perspective. International Journal of Engineering and Innovative Technology (IJEIT), 3 (1).

Rosenbaum, D. L., Clark, M. H., Convertino, A. D., Call, C. C., Forman, E. M., \& Butryn, M. L. (2018). Examination of nutrition literacy and quality of self-monitoring in behavioral weight loss. Annals of Behavioral Medicine, 52(9), 809-816.

Salah, H., MacIntosh, E., \& Rajakulendran, N. (2014). MaRS Market Insights. Wearable Tech: Leveraging Canadian Innovation to Improve Health. Retrieved April 12, 2017, from www.marsdd.com/news-insights/mars-reports/.

Sconce, S. (2016). Best practices to address security concerns with employee wearable technologies. (Applied Information Management Master of Science thesis). The Graduate School of the University of Oregon.

Seidell, J.C., \& Halberstadt, J. (2015). The Global Burden of Obesity and the Challenge of Prevention. Ann Nutr Metab., 66(1.2), 77-12.

Spann, S. (2016). Wearable Fitness Devices: Personal Health Data Privacy in Washington State. Seattle University Law Review, 39, 1411-1432.

The Heart and Stroke Foundation. (2016). National obesity week: South Africa's weighty problem. Retrieved on April 12, 2017, from http://www.heartfoundation.co.za/mediareleases/national-obesity-week-south-africa's-weighty-problem

Weng, G.S., Zailania, S., Iranmanesh, M., \& Hyun, S.S. (2017). Mobile taxi booking application service's continuance usage intention by users. Transportation Research Part D. 57, 207-216

Westin, A.F. (1967). Privacy and Freedom. New York: Atheneum.

World Health Organization, WHO. (2016). Obesity and overweight fact sheet. Retrieved April 17, 2017, from http://www.who.int/mediacentre/factsheets/fs311/en/

https://www.grandviewresearch.com/industry-analysis/digital-patient-monitoring-devices-market

Zheng, J., Shen, Y., Zhang, Z., Wu, T., Zhang, G., \& Lu, H. (2013). Emerging wearable medical devices towards personalized healthcare. Proceedings of the 8th International Conference on Body Area Networks, 30 September - 02 October, 2013. Boston, Massachusetts, USA (1). doi:10.4108/icst.bodynets.2013.253725. 